Check for updates

Cite this: Chem. Commun., 2017, 53,5416

Received 30th March 2017 , Accepted 20th April 2017

DOI: $10.1039 / c 7 c c 02453 c$

rsc.li/chemcomm

\section{A Co-based single-molecule magnet confined in a barium phosphate apatite matrix with a high energy barrier for magnetization relaxation $\dagger$}

\author{
Pavel E. Kazin, (D)*a Mikhail A. Zykin, ${ }^{b}$ Lev A. Trusov, ${ }^{b}$ Artem A. Eliseev, ${ }^{b}$ \\ Oxana V. Magdysyuk, ${ }^{c}$ Robert E. Dinnebier, (D) ${ }^{d}$ Reinhard K. Kremer, ${ }^{d}$ \\ Claudia Felser ${ }^{e}$ and Martin Jansen ${ }^{\text {de }}$
}

An apatite-type barium phosphate with a high content of cobalt ions in the trigonal channels features slow relaxation of magnetization with an energy barrier $U_{\text {eff }}$ of up to $387 \mathrm{~cm}^{-1}$, which is well above the values for all so far known d-metal based single-molecule magnets (SMMs).

Consisting of an atomic group with only one paramagnetic atom in a highly anisotropic environment, a mononuclear SMM also named a single-ion magnet (SIM) may perform as a molecule-size permanent magnet which reveals quantum behavior and may serve as a tiniest element in ultrahigh-density magnetic memory, molecular electronic, and spintronic devices. ${ }^{1}$ In order to enable its application, materials exhibiting higher blocking temperatures $\left(T_{\mathrm{b}}\right)$ and hence higher energy barriers $\left(U_{\text {eff }}\right)$ for magnetization reversal are required. These features are connected to huge singleion magnetic anisotropy arising from an efficient spin-orbital coupling (SOC) acting in an anisotropic (axial) crystal field. ${ }^{2}$ So far, the highest $U_{\text {eff }}$ values exceeding $1000 \mathrm{~cm}^{-1}$ have been encountered in complexes of rare-earth elements, however $T_{\mathrm{b}}$ did not scale up accordingly due to non-thermal relaxation pathways. ${ }^{3,4}$ Compounds of d-elements commonly exhibit lower $U_{\text {eff }}$ values: among the largest are $246 \mathrm{~cm}^{-1}$ for a linear coordinated $\mathrm{Fe}^{1+}$ methanide ${ }^{5}$ and $180 \mathrm{~cm}^{-1}$ for a $\mathrm{Co}^{2+}$ clathrochelate, ${ }^{6}$ both representing Kramers' ions with a $\mathrm{d}^{7}$ configuration. Recent magnetization studies on $\mathrm{Li}_{3-x} \mathrm{Fe}_{x} \mathrm{~N}$ have suggested that the linear coordinated $\mathrm{Fe}^{1+}$ ion also behaves like a SIM with

\footnotetext{
${ }^{a}$ Department of Chemistry, Lomonosov Moscow State University,

119991 Moscow, Russia. E-mail: kazin@inorg.chem.msu.ru; Tel: + 74959393440

${ }^{b}$ Department of Materials Science, Lomonosov Moscow State University,

119991 Moscow, Russia

${ }^{c}$ Diamond Light Source Ltd, Harwell Science and Innovation Campus, OX11 ODE Didcot, UK

${ }^{d}$ Max Planck Institute for Solid State Research, Heisenbergstrasse 1, 70569 Stuttgart, Germany

${ }^{e}$ Max Planck Institute for Chemical Physics of Solids, Nöthnitzer Str. 40, 01187 Dresden, Germany

$\dagger$ Electronic supplementary information (ESI) available: Experimental details, powder X-ray diffraction patterns, magnetic and relaxation data plots, crystal structure data, modeling and fitting data. See DOI: $10.1039 / \mathrm{c} 7 \mathrm{cc} 02453 \mathrm{c}$
}

$U_{\text {eff }}=300 \mathrm{~cm}^{-1} \cdot{ }^{7} \mathrm{Co}^{2+}$ is of special interest as it is common in stable compounds and may keep a large orbital moment in a crystal field so that a maximal value of magnetic anisotropy is achieved. ${ }^{8}$ Recently, we have found that small magnetically anisotropic atomic groups - dioxometallate anions - inserted into the channels of an apatite lattice revealed SIM features: the paramagnetic linear $[\mathrm{OCuO}]^{-}$-anions show $U_{\text {eff }}$ values of up to ca. $100 \mathrm{~cm}^{-1}, 9,10$ while for the bent $[\mathrm{OCoO}]^{2-}$-anions values of 51-59 $\mathrm{cm}^{-1}$ have been recorded. ${ }^{11}$ Remarkably, at a low Co concentration, a part of the magnetization exhibits a new very slow relaxation (VSR) process with an unusually high $U_{\text {eff }}$ of 200-275 $\mathrm{cm}^{-1}$. The precise nature of that remained unclear though. Since the most promising barium compound contained only $0.04 \mathrm{~mol}$ Co per formula unit and only $30 \%$ of its magnetization relaxed that way, we have made further efforts in optimizing these numbers. Indeed, we have managed to insert a large quantity of Co ions into the channels of the apatite-type barium phosphate. Here we present the magnetic properties of the new materials obtained, demonstrating VSR of the major part of the magnetization with $U_{\text {eff }}$ values considerably exceeding all those found before for open d-shell transition elements.

Samples with nominal composition $\mathrm{Ba}_{10}\left(\mathrm{PO}_{4}\right)_{6}\left(\mathrm{Co}_{x} \mathrm{OH}_{1-2 x}\right)_{2}$, where $x=0.05(\mathbf{1}), 0.3(2,2 \mathrm{a})$, and $0.5(3)$, were prepared as described in the ESI. $\dagger$ The final annealing was conducted at $1450{ }^{\circ} \mathrm{C}$ for 1 and 2 and $1400{ }^{\circ} \mathrm{C}$ for $2 \mathrm{a}$ and 3 . According to XRD the samples represent an apatite phase sometimes with a small admixture of $\mathrm{Ba}_{3}\left(\mathrm{PO}_{4}\right)_{2}$ and $\mathrm{CoO}$ (Fig. S1-S4, ESI $\dagger$ ). SEM/EDX of 3 reveals a polycrystalline barium phosphate apatite matrix containing Co in a quantity corresponding to $x \approx 0.3$, and $\mathrm{CoO}$ as micron-sized rounded grains embedded.

The crystal structure details of 3 were determined by Rietveld refinement of the synchrotron powder XRD pattern (the Diamond Light Source, United Kingdom, Fig. S1 and Tables S1-S3, ESI $\dagger) .+$ The compound crystallizes in the apatite structure, space group $P 6_{3} / m, a=10.1926(1), c=7.7532(1) \AA$. A Co-atom is located in the trigonal channel with the refined site occupancy corresponding to $x=0.33$, and is randomly displaced from the ideal $(0,0,0)$ position within the $a b$-plane (Fig. 1). Due to the two short 


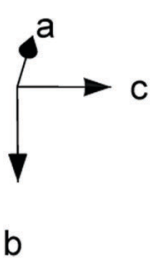

Fig. 1 A fragment of the trigonal channel (face-sharing octahedra of $\mathrm{Ba}$ atoms extending along the $c$-axis) in the apatite structure of the as prepared compound with the refined composition of $\mathrm{Ba}_{10}\left(\mathrm{PO}_{4}\right)_{6}\left(\mathrm{CO}_{0.33} \mathrm{O}_{0.72}\right)_{2}$.

contacts of Co with intra-channel oxygen atoms, a bent [OCoO] atomic group results, resembling the one found in the Co-doped strontium apatite. ${ }^{11,12}$ Since further $\mathrm{Co}-\mathrm{O}$ distances are greater than $3 \AA$, Co displays a coordination number of two. When the $[\mathrm{OCoO}]$ anion represents a separate unit in the crystal lattice, the possible Co-Co interatomic distances cannot be markedly below the values of the cell parameters. This suggests considering [OCoO] as a paramagnet which is well diluted in a diamagnetic matrix and is not involved in appreciable exchange and dipole interactions. The total content of Co in the sample, estimated from the XRD quantities of the apatite and $\mathrm{CoO}$ phases, corresponds to $x=0.47$ which is close to the nominal $x=0.5$. This confirms that the major amount of Co diluted in the apatite phase is found in the trigonal channels. All other samples were analyzed using XRD (Bruker D8 diffractometer, CuK $\alpha 1$ radiation). The powder XRD patterns of 2 and $2 \mathrm{a}$ were refined with somewhat lower accuracy, though still allowing us to localize a Co-atom near $(0,0,0)$ with the occupancy corresponding to $x=0.23$ (Fig. S2, S3 and Tables S4, S5, ESI†). A small amount of Co has not entered into the apatite phase and was detected as a $\mathrm{CoO}$ impurity (0.3-0.4 wt\%). The powder XRD pattern of 1 represented a pure apatite phase (Fig. S4, ESI $\dagger$ ). An observed electron density around $(0,0,0)$ could be attributed to intrachannel Co, but its (low) content was not estimated reliably (Table S6, ESI†).

In a previous communication for Co-doped strontium apatites,${ }^{11}$ we distinguished three magnetization relaxation regimes contributing to the total magnetization: (i) a field induced "normal" slow relaxation (NSR) with a $U_{\text {eff }}$ of tens of $\mathrm{cm}^{-1}$, covering the major fraction of the magnetization; (ii) a zero field very slow relaxation (VSR) with a $U_{\text {eff }}$ of some two hundreds of $\mathrm{cm}^{-1}$, mostly as a small contribution, and (iii) a paramagnetic behavior of a part of magnetization designated as a fast relaxation (FR). In the Co-doped barium apatites presented here, the frequency dependence of the ac susceptibility $\chi_{\mathrm{ac}}(f)$ measured under a zero dc field and above $10 \mathrm{~K}$ reveals a large $\chi^{\prime \prime}(T)$-peak connected to VSR (Fig. S5-S8, ESI $\dagger$ ). By simultaneously fitting $\chi^{\prime}(f)$ and $\chi^{\prime \prime}(f)$ in the generalized Debye model ${ }^{13}$ we determined a relaxation time $\tau$, its distribution width $\alpha$, and a fraction of VSR susceptibility $F$ (ESI $\dagger$ ). An adiabatic (high-frequency) susceptibility value was considered as an FR fraction as soon as we did not observe an NSR checking $\chi_{\mathrm{ac}}(f)$ in a magnetic field at low temperatures. The VSR fraction covers the major part of the ac susceptibility and at

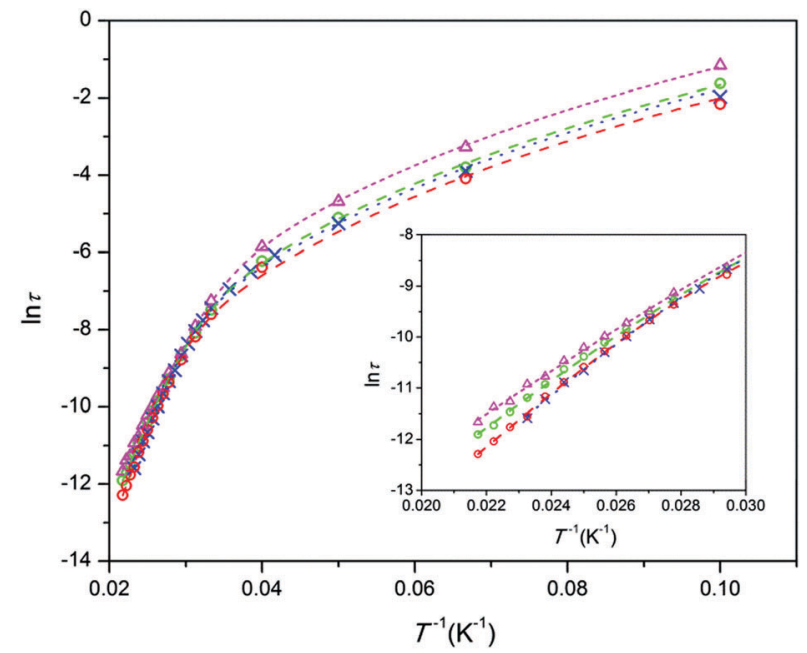

Fig. 2 Arrhenius plot for the relaxation time $\tau$ calculated from $\chi_{\text {ac }}(f)$ measured under a zero dc field; symbols - experimental data, lines - linear fits. Inset: An enlarged area at high temperatures. Sample designation: 1 - triangles, $\mathbf{2}$ - green circles, $\mathbf{2 a}$ - red circles, and $\mathbf{3}$ - crosses.

$10 \mathrm{~K}$ comprises $68,89,77$, and $74 \%$ for $\mathbf{1}, \mathbf{2}$, 2a, and 3 respectively. At $10 \mathrm{~K}, \alpha$ is relatively large, and amounts to 0.3-0.5. However, above $30 \mathrm{~K}, \alpha$ drops below 0.1 , implying a very narrow relaxation time distribution width which distinguishes SMMs.

The relaxation time values are depicted as Arrhenius-type plots in Fig. 2. At low temperatures, $\tau$ is limited by quantum tunneling and Raman processes. ${ }^{14}$ At higher temperatures, the dependence becomes steep and approaches a linear one, indicating that a thermally activated Orbach process is predominant. In the whole temperature range, the data can be fitted with the function $^{11,14}$

$$
\tau^{-1}=\tau_{\mathrm{QTM}}{ }^{-1}+B T+C T^{n}+\tau_{0}{ }^{-1} \exp \left(-U_{\text {eff }} / k_{\mathrm{B}} T\right),
$$

where $\tau_{\mathrm{QTM}}$ is the relaxation time of the quantum tunneling process, $B=A H^{m}$ ( $H$-magnetic field strength) is the coefficient of the direct process, $C$ is the coefficient of the Raman process, and $\tau_{0}$ is the attempt time for the thermally activated process. We adopted $B=0$ for the zero-field measurements and $\tau_{\mathrm{QTM}}{ }^{-1}=0$ as this process had a negligible effect on the fitting in the considered temperature interval. A good fitting was achieved with $n=5$ (which earlier was successfully applied for $\mathrm{Fe}^{1+}$ and $\mathrm{Co}^{2+}$ with a d $^{7}$ configuration $\left.{ }^{5,11}\right)$. The fitted parameters are: $U_{\text {eff }}=303(4)$, $338(5), 387(11)$, and $386(10) \mathrm{cm}^{-1} ; \tau_{0}=7.3(11) \times 10^{-10}, 1.9(3) \times$ $10^{-10}, 2.7(10) \times 10^{-11}$, and $2.6(10) \times 10^{-11} \mathrm{~s}, C=3.3(1) \times 10^{-5}$, $5.3(1) \times 10^{-5}, 7.4(3) \times 10^{-5}$, and $6.0(2) \times 10^{-5} \mathrm{~s}^{-1} \mathrm{~K}^{-5}$, for $1,2,2 \mathrm{a}$, and 3 respectively. One recognizes the tendency of $U_{\text {eff }}$ to increase with $x$ and with decreasing annealing temperature. However, $\tau_{0}$ values and even $\tau$ values at highest temperatures are in the opposite order to the $U_{\text {eff }}$ values. The reason for this is not clear so far and it is not excluded that the determined lower $U_{\text {eff }}$ values are underestimated in case the Orbach process does not dominate. In any case the maximum $U_{\text {eff }}$ obtained exceeds the highest one for $3 \mathrm{~d}-$-metal complexes ${ }^{5}$ ever observed by $50 \%$ and that of $\mathrm{Li}_{3-x} \mathrm{Fe}_{x} \mathrm{~N}^{7}$ by $30 \%$. Large $U_{\text {eff }}$ values strongly suggest 


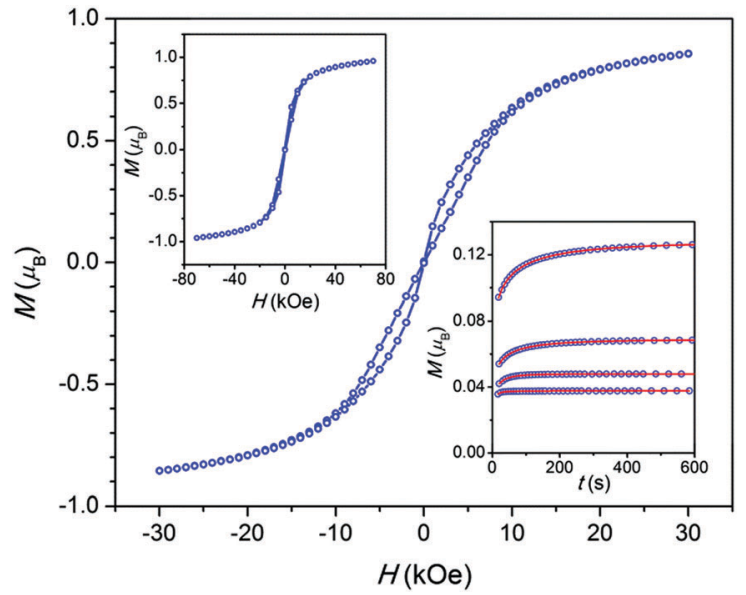

Fig. 3 Sample 3 . Field dependence of the magnetization per atom of $\mathrm{Co}$ at $T=1.8 \mathrm{~K}$ measured with a field sweeping rate of $50 \mathrm{Oe} \mathrm{s}^{-1}$. Top-left inset: The same in a larger field interval and at a field sweeping rate of $175 \mathrm{Oe} \mathrm{s}^{-1}$. Lines have been drawn to guide the eye. Bottom-right inset: Magnetization decay after switching on a field of $1.5 \mathrm{kOe}$ at temperatures of $2,4,6$, and $8 \mathrm{~K}$ (curves from top to bottom, respectively), symbols experimental points, lines - fitting.

that an orbital moment directly contributes to an electron spin due to a first order SOC.

The field dependence of a magnetization $M(H)$ at $1.8 \mathrm{~K}$ for all the samples (Fig. 3 and Fig. S9, ESI $\dagger$ ) shows an irreversibility which is relatively pronounced in intermediate fields, considerably weakened near the zero field, and becomes almost reversible in high fields. This behavior is usually explained considering a direct relaxation process strongly prevailing under high fields and a quantum tunneling of magnetization which is substantial in the zero field and is suppressed when a field is applied. ${ }^{1,14}$ In accordance with the latter, the dc magnetization decay measured for 3 under a field of 1.5 kOe follows an expanded exponential behavior (Fig. 3 inset) with $\tau$ ranging from 44 to $3 \mathrm{~s}$ in the $2-8 \mathrm{~K}$ temperature interval, while under the zero field the decay is too fast to be registered in a dc mode.

The electronic and even oxidation states of a Co-ion in a VSR-center are not yet clear due to the inconclusive results of XANES and red-ox titration. ${ }^{11}$ In order to clarify these items we will further analyze in detail the dc magnetic properties of 2, which has the highest VSR fraction, taking into consideration the distribution of Co in the sample.

In $2,74 \%$ of the Co resides in the trigonal channels of an apatite phase, $c a$. $15 \%$ as a $\mathrm{CoO}$ admixture, and the remainder is not assessed. $\mathrm{CoO}$ is an antiferromagnet with $T_{\mathrm{N}}=289 \mathrm{~K}$ and its $\chi$ slightly decreases from 0.005 to $0.0043 \mathrm{emu} \mathrm{mol}^{-1}$ with temperature ranging from $T_{\mathrm{N}}$ to $4.2 \mathrm{~K} .^{15}$ Therefore its contribution to the low temperature magnetization is negligibly small (below $0.2 \%$ ).

First, we have to subtract the FR contribution $(11 \%$ of the susceptibility at $10 \mathrm{~K}$ ). For this purpose, we synthesized a sample of barium apatite $\mathbf{4}$ with a composition different from that of 1 only by a slight excess (4\%) of Ba. The sample was finally annealed at $1300{ }^{\circ} \mathrm{C}$ in oxygen (as for $x=0.02$ we showed that oxygen annealing caused the VSR to disappear so that only FR stayed $\left.{ }^{11}\right) .4$ represents practically the pure apatite phase and

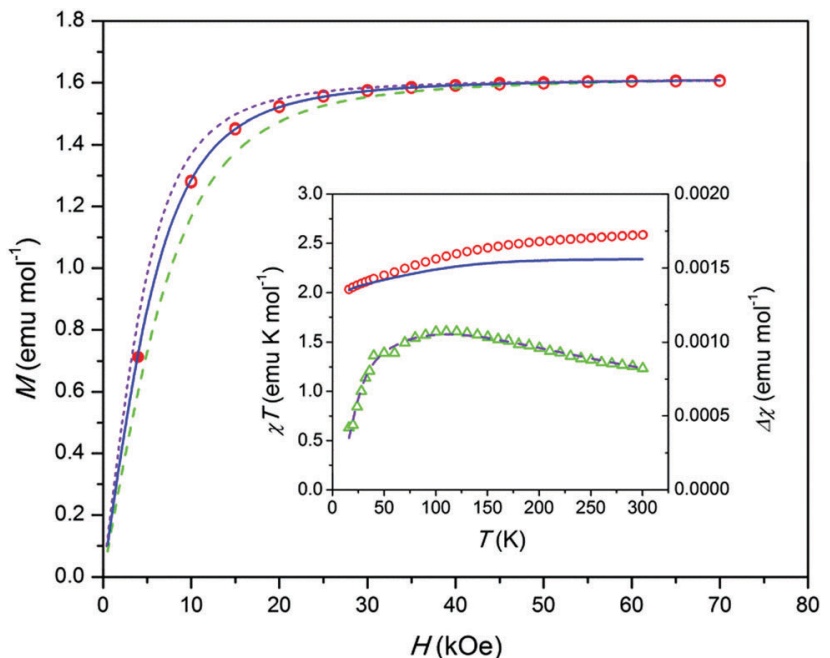

Fig. 4 Sample 2. Magnetization $M$ corrected for an FR contribution and normalized to $1 \mathrm{~mol}$ of Co vs. magnetic field $H$ at $T=1.8 \mathrm{~K}$. Open symbols data from the reversible part of $M(H)$, filled symbols - from an equilibrium dc value after the relaxation for 2 hours. Lines - modeling; dashed $-g_{z}=8$, solid $g_{z}=10$, short dashed $-g_{z}=12$. Inset: Left axis, product $\chi T$ corrected as above vs. temperature $T$. Circles - experimental data, solid line - modeling. Right axis, difference $\Delta \chi$ between the experimental and modeled $\chi$ vs. T. Triangles experiment, dashed line - fitting as described in the text and ESI. $\dagger$

exhibits only FR behavior (see Fig. S10 and S11, ESI $\dagger$ ). The dc susceptibility follows the Curie-Weiss law with $\theta=-3.2 \mathrm{~K}$ and $\mu_{\text {eff }}=4.99 \mu_{\mathrm{B}}$, which is close to $4.9 \mu_{\mathrm{B}}$ for $S=2$, implying that the compound contains $\mathrm{Co}^{3+}$ with a high-spin $\mathrm{d}^{6}$ configuration (Fig. S12, ESI $\dagger$ ). Rietveld refinement does not show an appreciable electron density near $(0,0,0)$ where a Co atom would be found, while an abnormally low atomic displacement parameter for P suggests Co substitution on P sites (Table S7, ESI $\dagger$ ).

Comparing the susceptibilities at $10 \mathrm{~K}$ we found that $10 \%$ of Co ions in 2 belong to the paramagnetic centers observed in 4 . Using the $\chi T(T)$ and low-temperature $M(H)$ dependence measured for 2 and 4 (Fig. S13 and S14, ESI $\dagger$ ) we corrected the data of 2 (Fig. 4). The corrected $M(H)$ data at $1.8 \mathrm{~K}$ indicate the magnetization of VSR centers only.

According to a modeling, ${ }^{11}$ an electronic structure of [OCoO] compatible with the observed high $U_{\text {eff }}$ implies that the ground doublet has very high easy axis anisotropy with $g_{x}$ and $g_{y}$ close to zero. In this case the low-temperature $M(H)$ may be approximately described by a Brillouin function with a certain $g_{\mathrm{z}}$ and $S_{\text {eff }}=1 / 2$ and with a proper powder-averaging by a numerical integration. ${ }^{9}$ Then by fitting experimental data, one can get simultaneously both, $g_{\mathrm{z}}=2(L+2 S)$ and a content of paramagnetic centers. Our modeling shows that the corrected $M(H)$ data fit well to $g_{z}=10$ with a content of paramagnetic centers corresponding to $58 \%$ of Co in 2 (see Fig. 4). Since such a quantity of Co can be found only in the apatite trigonal channels, this supports the assumption ${ }^{11}$ that the VSR center stems from an intrachannel Co atom like an NSR center. The remaining magnetically not-assessed $32 \%$ of Co ions seem to be in non-magnetic states at $T=1.8 \mathrm{~K}$, e.g. as exchange-coupled ions or low-spin $\mathrm{Co}^{3+}$. To such ions, the $\mathrm{CoO}$ impurity obviously contributes. 
The VSR center, an intra-channel twofold coordinated Co atom, most probably adopts an oxidation state of +2 or +3 . Among appropriate ground electronic multiplets for the linear Co complex discussed in ref. 11 , only ${ }^{4} \Phi_{\mathrm{g}}\left(\mathrm{Co}^{2+}\right)$ may provide large enough zero-field splitting to justify the observed $U_{\text {eff }}$ values, also for a moderately bent [OCoO] group. By modeling using the program CONDON, ${ }^{16}$ with ${ }^{4} \Phi_{\mathrm{g}}$ as a starting point, and adjusting the ligand field parameters, we obtained a theoretical $\chi T(T)$ dependence for a bent [OCo $\left.{ }^{\mathrm{II}} \mathrm{O}\right]$ (Fig. 4 inset, solid line). It corresponds to an electronic state with a first excited doublet at an energy very close to an experimental $U_{\text {eff }}$ of $338 \mathrm{~cm}^{-1}$ and with a $g_{z}=10$ ground doublet (see the ESI $\dagger$ ). The calculated $\chi T$ values approach the experimental ones at low temperature, but fall below as the temperature increases. Such a behavior may be connected to the presence of a fraction of antiferromagnetically coupled Co ions which do not contribute to the magnetization at low temperature but add an increasing quantity at increasing temperatures due to the decoupling of spins (this also concerns the CoO impurity). This assumption is further supported by the analysis of the difference $\Delta \chi(T)$ between experimental and calculated $\chi(T)$. The $\Delta \chi(T)$ data show a behavior common for an antiferromagnetic exchange cluster with the $\Delta \chi$ maximum at an intermediate temperature (Fig. 4 inset, triangles). It fits well to a Heisenberg $S=3 / 2$ dimer and comprises $c a$. 13\% of Co ions in 2. The fitting includes also a temperature independent paramagnetic contribution $\chi_{\text {TIP. }}$ It may account for the $\mathrm{CoO}$ admixture, the value being underestimated though ( $\mathrm{ca} .4 \%$ of Co ions in 2). The proposed dimers imply that some Co atoms may form intra-channel groups [OCoOCoO], which is allowed by the size of the channel.

As a result for 2, the following picture emerges. A major fraction of Co ions situated in the trigonal channels form the VSR centers and a minor fraction of the Co ions form the exchangecoupled clusters (probably dimers). Together they comprise $71 \%$ of Co ions, the number approaching that (74\%) derived from the intra-channel crystallographic occupancy. The VSR center includes a moderately bent atomic group [OCoO] apparently different from an earlier identified NSR center, a dioxocobaltate(II) ion. However, the electronic structure modeling suggests that the VSR center contains $\mathrm{Co}^{2+}$. Hence one may suppose that only next atoms in the vicinity of the Co atom are different for these two types of paramagnets. For example, the VSR center may represent hypothetical oxoperoxocobaltate(II) or oxohydroxocobaltate(II) ions. Of course, this proposal needs further clarification. The FR centers may be attributed mostly to crystallographically non-assessed Co ions in oxidation state +3 .

In summary, by Co doping, two types of SIM with high concentration can be controllably realized in an apatite lattice: the first one with a moderate $U_{\text {eff }}^{11}$ and the second one with an exceptionally high $U_{\text {eff. }}$ As mentioned previously in the context of materials showing moderate $U_{\text {eff }},{ }^{11}$ upon further increase of the Co content, an ordered hexagonal array of SIMs might be formed on the apatite (001) crystal surface with the grid interspacing equal to the cell parameter $a$. Having in mind that the high- $U_{\text {eff }}$ SIMs are also located in the apatite channels and in a high concentration, the same attractive prospects apply for the latter. Noteworthily, $U_{\text {eff }}$ increases by a factor of 7 on going from the first to the second type SIM. This strongly enhances chances for this type of material to be a prototype for an atomic-scale magnetic memory.

The work was supported by the Russian Science Foundation (RSF) under Grant no. 16-13-10031. We thank Walter Schnelle and Eva Bruecher for help with magnetic measurements and Christine Stefani for the XRD measurements.

\section{Notes and references}

$\ddagger$ Further details of the crystal structure investigations may be obtained from the Fachinformationszentrum Karlsruhe, 76344 EggensteinLeopoldshafen, Germany (Fax: +49-7247-808-666; E-mail: crysdata@ fizkarlsruhe.de, http://www.fiz-karlsruhe.de/request for deposited data.html) on quoting the depository number CSD 432190.

1 S. Thiele, F. Balestro, R. Ballou, S. Klyatskaya, M. Ruben and W. Wernsdorfer, Science, 2014, 344, 1135-1138.

2 L. Ungur and L. F. Chibotaru, Phys. Chem. Chem. Phys., 2011, 13, 20086-20090.

3 Y.-S. Ding, N. F. Chilton, R. E. P. Winpenny and Y.-Z. Zheng, Angew. Chem., 2016, 128, 16305-16308.

4 D. N. Woodruff, R. E. P. Winpenny and R. A. Layfield, Chem. Rev., 2013, 113, 5110-5148.

5 J. M. Zadrozny, D. J. Xiao, J. R. Long, M. Atanasov, F. Neese, F. Grandjean and G. J. Long, Inorg. Chem., 2013, 52, 13123-13131.

6 A. A. Pavlov, Y. V. Nelyubina, S. V. Kats, L. V. Penkova, N. N. Efimov, A. O. Dmitrienko, A. V. Vologzhanina, A. S. Belov, Y. Z. Voloshin and V. V. Novikov, J. Phys. Chem. Lett., 2016, 7, 4111-4116.

7 A. Jesche, R. W. McCallum, S. Thimmaiah, J. L. Jacobs, V. Taufour, A. Kreyssig, R. S. Houk, S. L. Bud'ko and P. C. Canfield, Nat. Commun., 2014, 5, 3333.

8 I. G. Rau, S. Baumann, S. Rusponi, F. Donati, S. Stepanow, L. Gragnaniello, J. Dreiser, C. Piamonteze, F. Nolting, S. Gangopadhyay, O. R. Albertini, R. M. Macfarlane, C. P. Lutz, B. A. Jones, P. Gambardella, A. J. Heinrich and H. Brune, Science, 2014, 344, 988-992.

9 P. E. Kazin, M. A. Zykin, Y. V. Zubavichus, O. V. Magdysyuk, R. E. Dinnebier and M. Jansen, Chem. - Eur. J., 2014, 20, 165-178.

10 P. E. Kazin, M. A. Zykin, W. Schnelle, C. Felser and M. Jansen, Chem. Commun., 2014, 50, 9325-9328.

11 P. E. Kazin, M. A. Zykin, W. Schnelle, Y. V. Zubavichus, K. A. Babeshkin, V. A. Tafeenko, C. Felser and M. Jansen, Inorg. Chem., 2017, 56, 1232-1240.

12 P. E. Kazin, O. R. Gazizova, A. S. Karpov, M. Jansen and Y. D. Tretyakov, Solid State Sci., 2007, 9, 82-87.

13 S. M. J. Aubin, Z. Sun, L. Pardi, J. Krzystek, K. Folting, L.-C. Brunel, A. L. Rheingold, G. Christou and D. N. Hendrickson, Inorg. Chem., 1999, 38, 5329-5340.

14 M. Atanasov, J. M. Zadrozny, J. R. Long and F. Neese, Chem. Sci., 2013, 4, 139-156.

15 P. S. Silinsky and M. S. Seehra, Phys. Rev. B: Condens. Matter Mater. Phys., 1981, 24, 419-423.

16 H. Schilder and H. Lueken, J. Magn. Magn. Mater., 2004, 281, 17-26. 